\title{
LE PHYLUM DES ACANTHOCEPHALA
}

TROISIĖME NOTE

La classe des PALAEACANTHOCEPHALA (Meyer 1931) (à suivre)

\author{
Par Yves J. GolvaN
}

LISTE DES HOTES

\section{INVERTEBRES}

\section{Crustacés MALACOSTRACES}

IsOPODES.

Armadillidium vulgare: Prosthorhynchus formosus.

Asellus aquaticus: Acanthocephalus anguillae.

Acanthocephalus lucii.

Acanthocephalus nanus.

Acanthocephalus ranae.

Filicollis anatis.

Chiridotea entomon : Echinorhynchus gadi.

AMPHIPODES.

Amphithoe rubricata: Echinorhynchus gadi.

Calliopus rathkei : Echinorhynchus gadi.

Carinogammarus roesselii : Polymorphus minutus.

Gammarus lacustris : Polymorphus magnus.

Gammarus locusta: Acanthocephalus lucii.

Echinorhynchus clavula.

Pomphorhynchus laevis.

Gammarus pulex : Polymorphus minutus.

Hyalella azteca ( $=$ H. knickerbockeri) : Leptorhynchoides thecatus.

Pontoporeia affinis : Corynosoma semerme.

Echinorhynchus salmonis.

Pontoporeia femorata: Echinorhynchus gadi.

Pontoporeia hoyi : Echinorhynchus coregoni. 
Schizopodes.

Euphausia pellucida: Echinorhynchus corrugatus.

DÉCAPODES.

Astacus astacus : Filicollis anatis (hòte d'attente).

Polymorphus minutus (hôte d'attente).

Cambarus affinis: Polymorphus minutus (hôte d'attente).

Palemon squilla: Arhythmorhynchus roseus (hôte d'attente).

\section{Insectes ACRIDIENS}

Catantops quadratus: Centrorhynchus sp.

\section{VERTEBRES}

\section{Classe des cyclostomes}

Ordre des Petromyzoniformes.

Caspiomyzon wagneri : Corynosoma strumosum (H. d'A.).

Lampetra fluviatilis : Corynosoma semerme (H. d'A.).

Corynosoma strumosum (H. d'A.).

Lampetra japonica: Corynosoma semerme (H. d'A.).

Petromyzon sp. : Corynosoma strumosum (H. d'A.).

\section{Classe des poissons}

\section{CHONDRYCHTHYES}

Sous-classe des Selachii

Série des Pleurotremata.

Ordre des GalÉiformes.

Mustelus laevis : Bolbosoma vasculosum (H. d'A.).

Ordre des SQualiformes.

Acanthias vulgaris : Echinorhynchus (E.) gadi.

Série des Hypotremata.

Ordre des RAJiformes.

A) Fam. des Rajidae.

Raja asterias: Acanthocephalus incrassatus.

Raja clavata : Echinorhynchus (E.) gadi.

B) Fam. des Dasyatidae.

Dasyatis say: Rhadinorhynchus (R.) pristis. 
C) Fam. des Myliobatidae.

Myliobatis aquila: Echinorhynchus (s.l.) urniger.

OSTEICHTHYES

Sous-classe des Actinopterygii

Série des Chondrostei.

Ordre des Acipenseriformes.

Acipenser brevirostris: Echinorhynchus (E.) attenuatus. Acipenser glaber: Leptorhynchoides thecatus.

Acipenser ruthenus : Acanthocephalus anguillae.

Echinorhynchus (P.) clavula.

Leptorhynchoides plagicephalus.

Pomphorhynchus tereticollis.

Acipenser stellatus: Leptorhynchoides plagicephalus.

Acipenser rubicundus: Acanthocephalus anguillae.

Acipenser sturio: Echinorhynchus (E.) gadi.

Leptorhynchoides plagicephalus.

Pomphorhynchus laevis.

Pomphorhynchus tereticollis.

Huso huso: Acanthocephalus anguillae.

Corynosoma strumosum.

Leptorhynchoides thecatus.

Pomphorhynchus laevis.

Série des Holostei.

Ordre des Amiformes.

A) Fam. des Amiidae.

Amia clava: Leptorhynchoides thecatus.

B) Fam. des Lepisosteidae.

Lepisosteus platostomus : Leptorhynchoides thecatus.

Lepisosteus platyrhynchus : Leptorhynchoides thecatus.

Série des Teleostei.

Ordre des Clupéiformes.

I) S./O, des CLUPEOIDEI.

A) Fam. des Clupeidae.

Alosa alosa : Pomphorhynchus tereticollis.

Rhadinorhynchus (R.) pristis.

Brachymystax lenok: Echinorhynchus (P.) lenok.

Caspialosa caspica : Corynosoma strumosum.

Caspialosa kesslerii : Corynosoma strumosum (H. d'A.). 
Caspialosa braschnikovi : Corynosoma strumosum (H. d'A.).

Caspialosa schaposchnikowi: Corynosoma strumosum (H. d'A.).

Caspialosa volgaensis : Corynosoma strumosum.

Clupea harengus : Corynosoma semerme (H. d'A.).

Corynosoma strumosum (H. d'A.).

Echinorhynchus (P.) clavula.

Echinorhynchus (E.) gadi.

Echinorhynchus (M.) salmonis.

Serrasentis socialis (H. d'A.).

Clupea sprattus: Echinorhynchus (E.) gadi.

Sardina pilchardus : Rhadinorhynchus (R.) pristis.

Sardinella aurita : Rhadinorhynchus (R.) pristis.

B) Fam. des Engraulidae.

Engraulis encrasicholus: Telosentis exiguus.

II) S./O. des SALMONOIDEI

A) Fam. des Osmeridae.

Hypomesus olidus : Corynosoma strumosum (H. d'A.).

Osmerus eperlanus : Corynosoma semerme (H. d'A.).

Corynosoma strumosum (H. d'A.).

Echinorhynchus (E.) gadi.

Echinorhynchus (M.) salmonis.

Echinorhynchus (P.) clavula.

Pomphorhynchus tereticollis.

Echinorhynchus (s.l.) eperlani.

Osmerus lanceolatus : Corynosoma strumosum (H. d'A.).

Osmerus mordax : Corynosoma hadweni (H. d'A.).

Leptorhynchoides thecatus.

B) Fam. des Salmonidae.

Coregonus albula: Acanthocephalus lucii.

Corynosoma semerme (H. d'A.).

Echinorhynchus (M.) salmonis.

Coregonus clupeaformis : Echinorhynchus (M.) salmonis. Leptorhynchoides thecatus.

Coregonus fera: Corynosoma semerme (H. d'A.).

Corynosoma strumosum (H. d'A.).

Echinorhynchus (M.) salmonis.

Coregonus fera widegreni: Echinorhynchus (M.) salmonis. Pomphorhynchus laevis.

Coregonus lavaretus: Acanthocephalus anguillae. Acanthocephalus lucii.

Corynosoma strumosum (H. d'A.).

Echinorhynchus (M.) salmonis.

Echinorhynchus (M.) truttae. 
Coregonus maraena: Acanthocephalus lucii.

Coregonus muksun: Acanthocephalus anguillae.

Echinorhynchus (M.) salmonis.

Pomphorhynchus laevis.

Coregonus oxyrhynchus: Acanthocephalus lucii.

Echinorhynchus (M.) salmonis.

Coregonus sardinella: Echinorhynchus (M.) salmonis.

Corynosoma strumosum (H. d'A.).

Coregonus ussuriensis: Acanthocephalus amuriensis.

Coregonus wartmanni: Pomphorhynchus laevis.

Coregonus sp.: Acanthocephalus anguillae.

Echinorhynchus (M.) salmonis.

Cristivomer namaycush: Echinorhynchus (E.) leidyi.

Echinorhynchus (M.) salmonis.

Leptorhynchoides thecatus.

Oncorhynchus adonis: Acanthocephalus echigoensis.

Oncorhynchus gorbuscha: Echinorhynchus (E.) gadi.

Oncorhynchus keta: Acanthocephalus echigoensis.

Bolbosoma caenoforme (H. d'A.).

Echinorhynchus (E.) gadi.

Oncorhynchus kisutch: Echinorhynchus (E.) gadi.

Oncorhynchus mason: Echinorhynchus (E.) gadi.

Echinorhynchus (M.) salmonis.

Oncorhynchus nerka: Acanthocephalus echigoensis.

Bolbosoma caenoforme (H. d'A.).

Echinorhynchus (E.) gadi.

Oncorhynchus tschawytscha: Echinorhynchus (E.) gadi.

Echinorhynchus (M.) salmonis.

Plecoglossus altivelis: Acanthocephalus echigoensis.

Acanthocephalus opsariichthydis.

Echinorhynchus (E.) sp.

Prosopium cylindraceum quadrilaterale: Leptorhynchoides thecatus.

Salmo erythrinus: Echinorhynchus (M.) salmonis.

Echinorhynchus (M.) truttae.

Salmo gairdnerii : Leptorhynchoides thecatus.

Salmo hucho: Pomphorhynchus laevis.

Pomphorhynchus tereticollis.

Salmo irideus : Acanthocephalus anguillae.

Acanthocephalus echigoensis.

Echinorhynchus (M.) truttae.

Pomphorhynchus laevis.

Salmo ischchan: Echinorhynchus (M.) sevani. 
Salmo mykiss : Echinorhynchus (M.) salmonis.

Salmo salar: Acanthocephalus lucii.

Bolbosoma heteracanthe (H. d'A.).

Echinorhynchus (E.) gadi.

Echinorhynchus (P.) clavula.

Echinorhynchus (M.) salmonis.

Echinorhynchus (M.) truttae.

Pomphorhynchus laevis.

Pomphorhynchus tereticollis.

Salmo trutta: Acanthocephalus anguillae.

Corynosoma strumosum (H. d'A.).

Echinorhynchus (P.) clavula.

Echinorhynchus (M.) salmonis.

Echinorhynchus (M.) truttae.

Salvelinus alpinus : Corynosoma semerme (H. d'A.).

Corynosoma strumosum (H. d'A.).

Echinorhynchus (M.) salmonis.

Pomphorhynchus laevis.

Salvelinus fontinalis: Acanthocephalus anguillae.

Acanthocephalus lateralis (?).

Leptorhynchoides thecatus.

Pomphorhynchus laevis.

Salvelinus malma: Echinorhynchus (E.) leidyi.

Echinorhynchus (M.) salmonis.

Rhadinorhynchus miyagawai.

Salvelinus oquassa marstoni: Echinorhynchus (M.) salmonis.

C) Fam. des Thymallidae.

Thymallus thymallus: Acanthocephalus anguillae.

Acanthocephalus lucii.

Corynosoma semerme (H. d'A.).

Corynosoma strumosum (H. d'A.).

Echinorhynchus (P.) clavula.

Echinorhynchus (M.) salmonis.

Echinorhynchus (M.) truttae.

Pomphorhynchus laevis.

Thymallus sp.: Pomphorhynchus laevis.

III) S./O. des ELOPOIDEI

Fam. des Albulidae.

Albula vulpes: Illiosentis africanus.

IV) S./O. des ESOCOIDEI

A) Fam. des Esocidae.

Esox lucius : Acanthocephalus anguillae.

Ann. de Parasitologie, T. XXXVI, N 1-2. -1961. 
Acanthocephalus lucii.

Corynosoma semerme (H. d'A.).

Corynosoma strumosum (H. d'A.).

Echinorhynchus (E.) gadi (?).

Echinorhynchus (M.) salmonis.

Echinorhynchus (M.) truttae.

Echinorhynchus (P.) clavula.

Leptorhynchoides thecatus.

Pomphorhynchus laevis.

Esox masquinongy masquinongy: Leptorhynchoides thecatus. Pomphorhynchus bulbocolli.

Esox niger: Leptorhynchoides thecatus.

Esox reicherti: Acanthocephalus amuriensis.

Paracanthocephalus curtus.

Paracanthocephalus tenuirostris.

Esox reticulatus: Leptorhynchoides thecatus.

Esox vermiculatus: Leptorhynchoides thecatus.

B) Fam. des Umbridae.

Umbra limi: Leptorhynchoides thecatus.

V) S./O. des MORMYROIDEI.

Fam. des Mormyridae.

Gnathonemus cyprinoides : Rhadinorhynchus (M.) horridus.

VI) S./O. des NOTOPTEROIDEI.

Fam. des Hyodontidae.

Hyodon tergisus: Leptorhynchoides thecatus.

VII) S./O. des MYCTOPHOIDEI.

A) Fam. des Synodidae.

Synodus foetens: Pomphorhynchus tereticollis.

Serrasentis socialis (H. d'A.).

B) Fam. des Myctophiidae.

Arctoscopus japonicus : Echinorhynchus (E.) gadi.

C) Fam. des Aulopidae.

Aulopus filamentosus : Bolbosoma vasculosum (H. d'A.).

Ordre des Percopsiformes.

Percopsis omiscomaycus: Leptorhynchoides thecatus.

Ordre des Cypriniformes.

I) S./O. des CHARACOIDEI.

Fam. des Gharacidae.

Alestes dentex: Paragorgorhynchus albertianus. 
Brycon hilarii : Echinorhynchus gracilis.

Hoplegnathus fasciatus: Longicollum pagrosomi.

Hydrocyon forskali: Pragorgorhynchus albertianus.

Mylina sp.: Echinorhynchus gomesi.

Mylosoma paraguayensis: Echinorhynchus (?) salobrensis.

Piaractus brachypomus : Echinorhynchus (M.) jucundus.

II) S./O. des CYPRINOIDEI.

A) Fam. des Gyprinidae.

Abramis ballerus: Acanthocephalus anguillae.

Pomphorhynchus laevis.

Abramis brama: Acanthocephalus anguillae.

Acanthocephalus lucii.

Corynosoma strumosum (H. d'A.).

Echinorhynchus (Metechinorhynchus) salmonis.

Echinorhynchus (Pseudechinorhynchus) clavula.

Pomphorhynchus laevis.

Abramis chrysoleucas: Pomphorhynchus bulbocolli.

Abramis sapa: Acanthocephalus anguillae.

Pomphorhynchus laevis.

Alburnus alburnus: Acanthocephalus anguillae.

Pomphorhynchus laevis.

Alburnus bipunctatus: Acanthocephalus anguillae.

Pomphorhynchus laevis.

Aspius aspius: Acanthocephalus anguillae.

Acanthocephalus lucii.

Corynosoma strumosum (H. d'A.).

Pomphorhynchus laevis.

Barbus avernensis : Pomphorhynchus laevis.

Barbus barbus: Acanthocephalus anguillae.

Acanthocephalus lucii.

Pomphorhynchus laevis.

Barbus brachycephalus: Pomphorhynchus laevis.

Barbus capito: Pomphorhynchus laevis.

Barbus goktschaikus : Pomphorhynchus laevis.

Barbus plebejus : Pomphorhynchus laevis.

Barbus sp. : Acanthocephalus lucii.

Blicca bjorkna: Acanthocephalus anguillae.

Acanthocephalus lucii.

Corynosoma semerme (H. d'A.).

Echinorhynchus (Metechinorhynchus) salmonis.

Pomphorhynchus laevis.

Carassius carassius: Acanthocephalus anguillae. Acanthocephalus minor. 
Arhythmorhynchus hispidus (H. d'A.).

Rhadinorhynchus (Rhadinorhynchus) exilis (?).

Carassius carassius auratus: Paracanthocephalus tenuirostris. Rhadinorhynchus (Rhadinorhynchus) exilis (?).

Chalcalburnus chalcoides : Corysonoma strumosum (H. d'A.). Pomphorhynchus laevis.

Chanodichthys macropus: Micracanthorhynchina motomurai.

Chondrostoma nasus : Pomphorhynchus laevis.

Cyprinus carpio: Acanthocephalus anguillae.

Acanthocephalus lucii.

Acanthocephalus opsariichthydis.

Echinorhynchus (Pseudechinorhynchus) clavula.

Leptorhynchoides thecatus.

Paracanthocephalus curtus.

Paracanthocephalus tenuirostris.

Pomphorhynchus bulbocolli.

Pomphorhynchus laevis.

Cyprinus orbula: Pomphorhynchus laevis.

Diptychus dybowskyi : Pomphorhynchus laevis.

Elopichthys bambusa: Paracanthocephalus tenuirostris.

Erythroculter erythropterus : Echinorhynchoïdes dogieli.

Paracanthocephalus curtus.

Paracanthocephalus tenuirostris.

Gobio aphya : Acanthocephaloides propinquus.

Gobio elongatus : Acanthocephalus opsariichthydis.

Gobio gobio : Acanthocephalus anguillae.

Acanthocephalus lucii.

Echinorhynchus (Pseudechinorhynchus) clavula.

Echinorhynchus (Pseudechinorhynchus) cinctulus (?).

Pomphorynchus laevis.

Gnathopogon elongatus : Acanthocephalus opsariichthydis.

Hemibarbus barbus: Acanthocephalus opsariichthydis.

Hemibarbus labeo : Acanthocephalus parallelotestis. Echinorhynchoïdes dogieli.

Hybopsis storerianus : Leptorhynchoïdes thecatus.

Idus idus : Acanthocephalus anguillae.

Acanthocephalus lucii.

Corynosoma semerme (H. d'A.).

Corynosoma strumosum (H. d'A.).

Echinorhynchus (Pseudechinorhynchus) clavula.

Echinorhynchus (Pseudechinorhynchus) cinctulus (?).

Pomphorhynchus laevis.

Leuciscus agassizi : Pomphorhynchus laevis. 
Leuciscus cavedanus : Pomphorhynchus laevis.

Leuciscus cephalus : Acanthocephalus anguillae.

Corynosoma strumosum (H. d'A.).

Pomphorhynchus laevis.

Leuciscus hakonensis: Acanthocephalus opsariichthydis.

Leuciscus idbarus: Acanthocephalus anguillae.

Echinorhynchus (Metechinorhynchus) salmonis.

Pomphorhynchus laevis.

Leuciscus illyricus : Acanthocephalus lucii.

Pomphorhynchus laevis.

Leuciscus leuciscus : Echinorhynchus (Metechinorhynchus) salmonis.

Echinorhynchus (Pseudechinorhynchus) clavula.

Pomphorhynchus laevis.

Polymorphus minutus (H. d'A.).

Leuciscus rutilus : Acanthocephalus anguillae.

Acanthocephalus lucii.

Corynosoma strumosum (H. d'A.).

Polymorphus minutus (H. d'A.).

Leuciscus schmidti: Pomphorhynchus laevis.

Pomphorhynchus perforator.

Leuciscus waleckii : Bolbosoma sp.

Hemirhadinorhynchus leuciscus.

Paracanthocephalus tenuirostris.

Leuciscus sp.: Acanthocephalus amuriensis. Arhythmorhynchus frassoni (H. d'A.).

Opsariichthys uncirostris: Acanthocephalus opsariichthydis.

Parabramis pekinensis : Paracanthocephalus tenuirostris. Pseudorhadinorhynchus markewitchii.

Pelecus cultratus : Acanthocephalus anguillae. Echinorhynchus (s. 1.) peleci.

Phoxinus phoxinus : Acanthocephalus lucii.

Pomphorhynchus laevis.

Polymorphus minutus (H. d'A.).

Phoxinus steindachneri: Acanthocephalus opsariichthydis.

Pseudaspius leptocephalus : Paracanthocephalus tenuirostris. Pseudorhadinorhynchus markewitchii.

Pseudorhadinorhynchus pseudaspii.

Pseudorasbora parva: Acanthocephalus opsariichthydis. Paracanthocephalus tenuirostris.

Rutilus rutilus : Acanthocephalus anguillae. Acanthocephalus lucii.

Corynosoma strumosum (H. d'A.).

Echinorhynchus (Metechinorhynchus) salmonis.

Echinorhynchus (Pseudechinorhynchus) clavula.

Pomphorhynchus laevis. 
Scardinius erythrophthalmus : Acanthocephalus anguillae.

Acanthocephalus lucii.

Echinorhynchus (Metechinorhynchus) salmonis.

Polymorphus minutus (H. d'A.).

Pomphorhynchus laevis.

Schizopygopsis koslovi: Echinorhynchus (Metechinorhynchus) alpinus.

Schizopygopsis stoliczkae: Acanthocephalus kashmiriensis.

Schizothorax pseudoaksaiensis issykkuli: Pomphorhynchus perforator.

Schizothorax sp.: Echinorhynchus (?) orientalis.

Semotilus a. atromaculatus : Leptorhynchoides thecatus.

Squalius cavedanus : Pomphorynchus laevis.

Tinca tinca: Acanthocephalus anguillae.

Acanthocephalus graciliacanthus.

Acanthocephalus lucii.

Pomphorhynchus laevis.

Varicorhinus capoeta sevangi: Echinorhynchus (Metechinorhynchus) baeri.

Pomphorhynchus kostylewi.

Pomphorhynchus laevis.

Vimba vimba: Acanthocephalus anguillae.

Acanthocephalus lucii.

Corynosoma strumosum (H. d'A.).

Echinorhynchus (Metechinorhynchus) salmonis.

Pomphorhynchus laevis.

Xenocypris macrolepis : Paracanthocephalus tenuirostris.

Zacco platypus : Acanthocephalus opsarichthydis.

Micracanthorhynchina motomurai.

Zacco temmincki : Micracanthorhynchina dakusuiensis.

Micracanthorhynchina motomurai.

Zacco sp. : Micracanthorhynchina motomurai.

B) Fam. des Gatostomidae.

Carpiodes carpio : Leptorhynchoides thecatus. Pomphorhynchus bulbocolli.

Catostomus commersoni : Leptorhynchoides thecatus. Pomphorhynchus bulbocolli.

Erimyzon s. sucetta: Leptorhynchoides thecatus.

Moxostoma rubreques : Leptorhynchoides thecatus.

C) Fam. des Cobitidae.

Hymenophysa curta: Acanthocephalus opsarichthydis.

Misgurnus anguillicaudatus : Acanthocephalus opsarichthydis. Echinorhynchus (M.) salmonis. 
Misgurnus fossilis: Tenuiproboscis misgurni.

Nemachilus barbatula: Acanthocephalus anguillae.

Acanthocephalus lucii.

Pomphorhynchus laevis.

Sakalinorhynchus skrjabini.

Nemachilus kashmiriensis : Pomphorhynchus kashmiriensis

III) S./O. des SILUROIDEI.

A) Fam. des Bagridae.

Ameiurus m. melas: Leptorhynchoides thecatus.

Pomphorhynchus bulbocolli.

Ameiurus n. natalis : Leptorhynchoides thecatus.

Pomphorhynchus bulbocolli.

Ameiurus n. nebulosus : Leptorhynchoides thecatus.

Pomphorhynchus bulbocolli.

Bagrus bayad: Paragorgorhynchus albertianus.

Chrysichthys sp. : Arhythmorhynchus siluricola (H. d'A.).

Galeichthys felis : Gorgorhynchus medius.

Gephyroglanis sp. : Arhythmorhynchus siluricola (H. d'A.).

Ictalurus lacustris punctatus : Leptorhynchoides thecatus.

Liocassis ussuriensis : Paracanthocephalus curtus.

Noturus flavus: Leptorhynchoides thecatus.

Pangasius pangasius : Mehrarhynchus prashadi.

Pilodictus olivaris : Leptorhynchoides thecatus.

Pseudobagrus fluvidraco: Paracanthocephalus tenuirostris.

B) Fam. des Schilbeidae.

Schilbe mystus : Paragorgorhynchus albertianus.

Schilbeodes mollis : Leptorhynchoides thecatus.

C) Fam. des Doradidae.

Doras niger: Echinorhynchus (s. 1.) impudicus.

D) Fam. des Siluridae.

Parasilurus asotus : Acanthocephalus amuriensis.

Acanthocephalus gotoi.

Acanthocephalus minor.

Acanthocephalus opsariichthydis.

Paracanthocephalus curtus.

Paracanthocephalus tenuirostris.

Pseudoechinorhynchus parasiluri.

Longicollum pagrosomi.

Silurus glanis: Acanthocephalus anguillae.

Acanthocephalus lucii.

Corynosoma strumosum (H. d'A.). 
Echinorhynchus (Pseudechinorhynchus) clavula. Echinorhynchus (Pseudechinorhynchus) cinctulus. Pomphorhynchus laevis.

E) Fam. des Plotosidae.

Plotosus anguillaris: Heterosentis plotosi.

Ordre des Anguilliformes.

I) S./O. des ANGUILLOIDEI.

Fam. des Anguillidae.

Anguilla anguilla: Acanthocephalus anguillae.

Acanthocephalus lucii.

Acanthocephaloides incrassatus.

Acanthocephaloides propinquus.

Corynosoma semerme (H. d'A.).

Corynosoma strumosum (H. d'A.).

Echinorhynchus (Echinorhynchus) gadi.

Echinorhynchus (Pseudechinorhynchus) clavula.

Echinorhynchus (Metechinorhynchus) salmonis.

Filicollis anatis (H. d'A.).

Polymorphus minutus (H. d'A.).

Pomphorhynchus laevis.

Pomphorynchus tereticollis.

Telosentis molini.

Anguilla bostoniensis : Leptorhynchoides thecatus.

Anguilla chrysypa: Acanthocephalus anguillae.

Echinorhynchus (Metechinorhynchus) salmonis.

Leptorhynchoides thecatus.

Anguilla japonica : Acanthocephalus gotoi.

Longicollum pagrosomi.

II) S./O. des CONGROIDEI.

Fam. des Gongridae.

Conger conger : Bolbosoma vasculosum (H. d'A.).

Corynosoma strumosum (H. d'A.).

Echinorhynchus (E.) gadi.

Echinorhynchus (s. l.) solitarius.

Conger myriaster: Echinorhynchus (E.) gadi.

Ordre des CyPRInodontiformes.

A) Fam. des Gyprinodontidae.

Fundulus chrysotus : Leptorhynchoides thecatus.

Fundulus diaphanus menona: Leptorhynchoides thecatus.

Fundulus dispar: Leptorhynchoides thecatus.

Fundulus grandis: Leptorhynchoides thecatus. 
Fundulus majalis: Leptorhynchoides thecatus.

Fundulus notatus: Leptorhynchoides thecatus.

Fundulus similis : Leptorhynchoides thecatus.

Orestias tschudii: Echinorhynchus (s. 1.) orestiae.

B) Fam. des Poeciliidae.

Gambusia affinis : Leptorhynchoides thecatus.

Gambusia affinis holbrooki: Leptorhynchoides thecatus.

Ordre des Gadiformes.

A) Fam. des Macruridae.

Coelorhynchus coelorhynchus : Echinorhynchus (s. 1.) urniger.

B) Fam. des Gadidae.

Boreogadus saidn: Echinorhynchus (Echinorhynchus) gadi.

Brosmius brosme: Echinorhynchus (Echinorhynchus) gadi.

Eleginus navaga: Echinorhynchus (Echinorhynchus) gadi.

Gadus aeglefinus: Echinorhynchus (Echinorhynchus) attenuatus. Echinorhynchus (Echinorhynchus) gadi.

Rhadinorhynchus (Rhadinorhynchus) pristis.

Gadus callarias (= Gadus morrhua): Acanthocephalus lucii (?).

Corynosoma semerme (H. d'A.).

Corynosoma strumosum (H. d'A.).

Echinorhynchus (Echinorhynchus) gadi.

Echinorhynchus (Pseudechinorhynchus) clavula (?).

Echinorhynchus (Metechinorhynchus) salmonis (?).

Bolbosoma vasculosum (H. d'A.).

Pomphorhynchus tereticollis.

Leptorhynchoides thecatus.

Rhadinorhynchus (Rhadinorhynchus) pristis.

Gadus cruentatus: Acanthocephaloides incrassatus.

Gadus macrocephalus : Corynosoma strumosum (H. d'A.). Echinorhynchus (Echinorhynchus) gadi.

Gadus minutus: Echinorhynchus (Echinorhynchus) gadi.

Gadus pollachius : Echinorhynchus (Echinorhynchus) gadi.

Gadus poutassou: Pomphorhynchus tereticollis.

Gadus virens: Echinorhynchus (Echinorhynchus) gadi.

Lota lota maculosa: Leptorhynchoides thecatus.

Lota vulgaris: Acanthocephalus anguillae.

Acanthocephalus lucii.

Corynosoma semerme (H. d'A.).

Corynosoma strumosum (H. d'A.).

Echinorhynchus (Echinorhynchus) gadi (?).

Echinorhynchus (Pseudechinorhynchus) cinctulus. 
Echinorhynchus (Pseudechinorhynchus) clavula. Pomphorhynchus laevis.

Lotella phycis : Echinorhynchus (Echinorhynchus) dissimilis. Echinorhynchus (Echinorhynchus) gadi.

Echinorhynchus (Echinorhynchus) lotellae.

Merlangus vulgaris: Echinorhynchus (Echinorhynchus) gadi. Pomphorhynchus tereticollis.

Merluccius vulgaris : Bolbosoma vasculosum (H. d'A.). Echinorhynchus (E.) gadi.

Merluccius sp. : Aspersentis johni.

Microgadus proximus : Echinorhynchus (Echinorhynchus) gadi.

Microgadus tamcod: Echinorhynchus (Echinorhynchus) gadi.

Molva byrkelange : Echinorhynchus (E.) gadi.

Odontogrammus euxinus : Acanthocephaloides incrassatus. Acanthocephaloides propinquus.

Onos cimbrius : Corynosoma semerme (H. d'A.). Corynosoma sp. (H. d'A.). Echinorhynchus (E.) gadi.

Onos mustela: Echinorhynchus (E.) gadi. Echinorhynchus (E.) leidyi.

Phycis mediterraneus : Bolbosoma vasculosum (H. d'A.).

Theragra chalcogramma: Echinorhynchus (Echinorhynchus) gadi.

Trisopterus minutus: Acanthocephaloides incrassatus. Echinorhynchus (Echinorhynchus) gadi.

Echinorhynchus (Pseudechinorhynchus) clavula.

Urophycis tenuis : Echinorhynchus (Echinorhynchus) gadi.

Ordre des LAMPRIDIFormes.

Fam. des Trachypteridae.

Trachypterus falx: Bolbosoma vasculosum (H. d'A.).

Regalecus glesne: Bolbosoma vasculosum (H. d'A.).

Ordre des Pleuronectiformes.

I) S./O. des PSETTODOIDEI.

Fam. des Psettodidae.

Psettodes belcheri: Serrasentis socialis (H. d'A.).

Psettodes erumei : Serrasentis chauhani.

II) S./O. des SOLEOIDEI.

A) Fam. des Cynoglossidae.

Cynoglossus canariensis : Serrasentis socialis (H. d'A.).

Cynoglossus zanzibarensis: Acanthocephaloides chabanaudi.

Symphurus urniger: Rhadinorhynchus (R.) pristis. 
B) Fam. des Soleidae.

Alaeops plinthus: Hypoechinorhynchus alaeopis.

Microchirus wittei: Acanthocephaloides propinquus.

Monochirus atlanticus : Acanthocephaloides propinquus.

Monochirus hispidus : Acanthocephaloides propinquus.

Solea cuneata: Serrasentis socialis (H. d'A.).

Solea hexophthalma: Serrasentis socialis (H. d'A.).

Solea impar: Acanthocephaloides soleae (?). Echinorhynchus (s.1.) rhytidodes.

Solea nasuta: Acanthocephaloides kostylewi.

Solea vulgaris: Acanthocephalus lucii (?). Acanthocephaloides incrassatus.

Acanthocephaloides propinquus.

Echinorhynchus (Echinorhynchus) gadi.

Pomphorhynchus tereticollis.

Echinorhynchus (s. l.) urniger.

(A suivre). 